\author{
${ }^{1 *}$ Voronkov V.V., ${ }^{2}$ Voronkova E.N. \\ ${ }^{1}$ Kazakh-British Technical University, Almaty, Kazakhstan \\ ${ }^{2}$ Al-Farabi Kazakh National University, Almaty, Kazakhstan \\ "e-mail: voronkov.v@hotmail.com
}

\title{
Investigation of Static Properties of Strongly Coupled Plasma with Molecular Dynamics Method
}

\begin{abstract}
Main criteria for creation of a code of molecular dynamics method for studying properties of single-particle systems with detemined interaction potential are defined in this paper. It is proved that application of these criteria allows us to write the code of molecular dynamics method which is in good agreement with the results obtained by other authors. The code of molecular dynamics method has been implemented in a software environment Wolfram Mathematica. The velocity Verlet algorithm and the Rahman method are used to determine dynamics of particles. With the help the code written in Wolfram Mathematica language it is demonstrated that short range symmetries appear in a strongly coupled plasma.

Key words: molecular dynamics, Rahman method, radial distribution functions, velocity Verlet algorithm.
\end{abstract}

\section{Introduction}

It is possible to describe the properties of a wide range of different objects ranging from space plasma to atomic nuclei by using single-particle approximation. In this paper we define criteria for writing a molecular dynamics code to study the properties of single-particle models. The essence of the molecular dynamics method is the application of classical laws of mechanics to determine the configuration of positions and velocities of particles of different systems. Compared with the Monte Carlo method and the method of numerical solution of a closed system of Ornstein-Zernike equations in hyperchain approximation, the main advantage of the method of molecular dynamics is the ability to study the changes in system configurations over time. For this purpose we created a code of molecular dynamics method in software environment Wolfram Mathematica. The advantage of Wolfram Mathematica is usage of various lowlevel programming languages, which greatly speeds up calculations, thereby improves the quality of research. At the same time Mathematica uses its own high-level programming language which enables a researcher to adequately describe such complex systems as dense plasma.
A typical software implementation of the algorithm of molecular dynamics includes the following steps:

1. Determination of macro- and micro parameters describing conditions of a computer simulation, such as pressure, temperature, numerical density, masses of different types of particles etc.

2. Determination of an initial configuration, which includes the generation of random coordinates and velocities of the particles. It is necessary to take into account restrictions imposed on the mutual positions of the particles and the laws of the velocity distribution for each type of particles. It must be ensured that the initial configuration does not include the superposition of particles on each other, unusual local concentrations, because it may result in too large values of forces and further instability of numerical integration. Initial distribution of velocities for non-degenerate systems can be obtained by means of Maxwell-Boltzmann distribution.

3. Correction of the initial velocity distribution of the particles to obtain zero total momentum vector of a particle system.

4. In this paper we use NVE-ensemble approach, where each cycle of calculations uses the adjustment of the particle velocities to ensure

Printed in Kazakhstan 
compliance with the law of conservation of energy of the system [1].

5. Calculation of the accelerations acting on each particle of the system. At this stage, it is important the correct choice of potentials of interparticle interactions characterizing the properties of the studied medium. In particular at high densities characteristic distances are getting smaller than the wavelength of de Broglie, which leads to appropriate adjustments in the interaction potentials.

6. Numerical integration of motion equations. Moreover, this stage of calculations significantly depends on the selected algorithm for determining equations of motion. In this work, we use the Velocity Verlet algorithm and Rahman method, which will be described below.

7. Repetition of steps $3-5$ until the desired time interval is obtained, in which there will be sufficient changes in the system configuration.

8. Recording of the configuration settings of the particle system on the hard disk for further statistical processing. In addition to the coordinates and velocities, particle accelerations can be recorded what depends on the selected algorithm for determining of the equations of motion.

9. Repetition of steps $6-8$ until the sufficient number of system configurations are accumulated that will allow us to produce statistical calculations with the required accuracy. Determining the number of repetitions of steps $6-8$, we need to take into account that the total estimated time in which we study the system of particles should be much longer than the typical relaxation times of the investigated physical quantities.

\section{The Velocity Verlet Algorithm}

In this paper we use the Velocity Verlet algorithm [2] that stands out for the precision and speed of calculations. In this method, we first calculate the forces acting on the particles, as derivatives of the interaction potentials:

$$
\vec{F}=-\vec{\nabla}_{i} \sum_{\mathrm{j}} \mathrm{V}\left(\mathrm{r}_{\mathrm{ij}}\right) .
$$

Then we calculate the new coordinates of the atoms that are used in finding of resultant forces:

$$
\vec{r}_{i}(t+\Delta t)=\vec{r}_{i}(t)+\vec{v}_{i}(t) \Delta t+\frac{\vec{a}_{i}(t) \Delta t^{2}}{2} .
$$

In the next step we determine the particle velocities, and take into account the accelerations at two points in time:

$$
\vec{v}_{i}(t+\Delta t)=\vec{v}_{i}(t)+\frac{\vec{a}_{i}(t)+\vec{a}_{i}(t+\Delta t)}{2} \Delta t .
$$

here $v_{r m s}$ - mean square velocity of particles, $\langle r\rangle$. the average distance between the particles.

Initial velocities of the particles are selected using a random number generator in accordance with the Maxwell distribution at a given temperature. Data on velocities and positions of the particles are regularly recorded on the hard disk of a computer to store for further calculations.

\section{The Rahman Method}

Firstly, the initial configuration of the coordinates $\vec{r}_{i}(t)$ and velocities of particles $\vec{v}_{i}(t)$ is also set randomly in the Rahman method [3]. Secondly, the accelerations of the particles $\vec{a}_{i}(t)$ are calculated for the same time step $\Delta t$ using the selected interaction potentials. Then the first approximation of the particle coordinates $\vec{r}^{(1)}{ }_{i}(t+\Delta t)$ is performed for the next time step $t+\Delta t$ :

$$
\vec{r}^{(1)}(t+\Delta t)=\vec{r}_{i}(t)+\vec{v}_{i}(t) \Delta t
$$

At the next stage, the calculation of the particle accelerations $\vec{a}_{i}(t+\Delta t)$ for time step $t+\Delta t$ is performed using the first approximation of the locations of particles $\vec{r}^{(1)}{ }_{i}(t+\Delta t)$, and then the velocities are also calculated:

$$
\vec{v}_{i}(t+\Delta t)=\vec{v}_{i}(t)+\frac{\vec{a}_{i}(t)+\vec{a}_{i}(t+\Delta t)}{2} \Delta t
$$

Coordinates of particles in the next approximation is computed by the formula using mean velocity values for the two time steps $t$ and и $t+\Delta t$ :

$$
\vec{r}_{i}^{(2)}(t+\Delta t)=\vec{r}_{i}(t)+\frac{\vec{v}_{i}(t)+\vec{v}_{i}(t+\Delta t)}{2} \Delta t .
$$

Steps 4-6 are repeated until the desired accuracy of the calculations is obtained. The advantage of this method is the high accuracy of the calculations, but this increased accuracy is achieved with relatively high cost of computer time. 


\section{The choice of time step $\Delta t$}

The correct choice of time step is very important in the molecular dynamics method. Computer calculation time is proportional to the number of time steps and therefore the larger $\Delta t$ the greater the amount of configurations will be computed. However, the accuracy of the integration of the equations of motion exponentially decreases with increasing time step $\Delta t$. Required accuracy for certain values $\Delta t$ can be achieved by using successive refinement algorithms (predictorcorrector) like in the Rahman method [3], but the consumption of computer time increases significantly, which devalues the increase of the time step. The following criterion of step selection in modeling of nonideal plasma is proposed in [4]:

$$
\Delta \mathrm{t}<<\frac{\mathrm{a}_{\mathrm{B}}}{\mathrm{V}_{\mathrm{T}}},
$$

Where $a_{B}$ - the first Bohr orbit radius of the electron in a hydrogen atom, $V_{T}$ - thermal velocity. From our experience of application of molecular dynamics method, it follows that the following criteria must be fullfilled:

$$
\Delta t<<\sqrt{\frac{2\langle r\rangle}{\langle a\rangle}},
$$

$$
\begin{gathered}
\Delta t<<\frac{\langle V\rangle}{\langle a\rangle}, \\
\Delta t<<\frac{\langle r\rangle}{\langle V\rangle} .
\end{gathered}
$$

Comparison of calculations for the selected step $\Delta \mathrm{t}$ with calculations for steps $1.5 \Delta \mathrm{t}$ and $2 \Delta \mathrm{t}$ can be regarded as a test of the correctness of the choice of the time step. The results of calculations have to be close to each other.

\section{Validating the Correctness of the Written Code}

One of the key characteristics of the singleparticle medium is a pair radial distribution function $\mathrm{g}(\mathrm{r})$, which is the probability density of the location of the second particle at a distance $r$ from the first one.

Calculations in paper [3] have been reproduced here to verify the correctness of the developed main criteria for implementation of the molecular dynamics code. Properties of liquid argon at temperature of $94.4 \mathrm{~K}$ and density of $1.374 \mathrm{~g} / \mathrm{cm}^{3}$ have been studied. Calculations were performed using the Rahman method and the Velocity Verlet algorithm. Both methods gave similar results. The following radial distribution function was obtained in [3]:

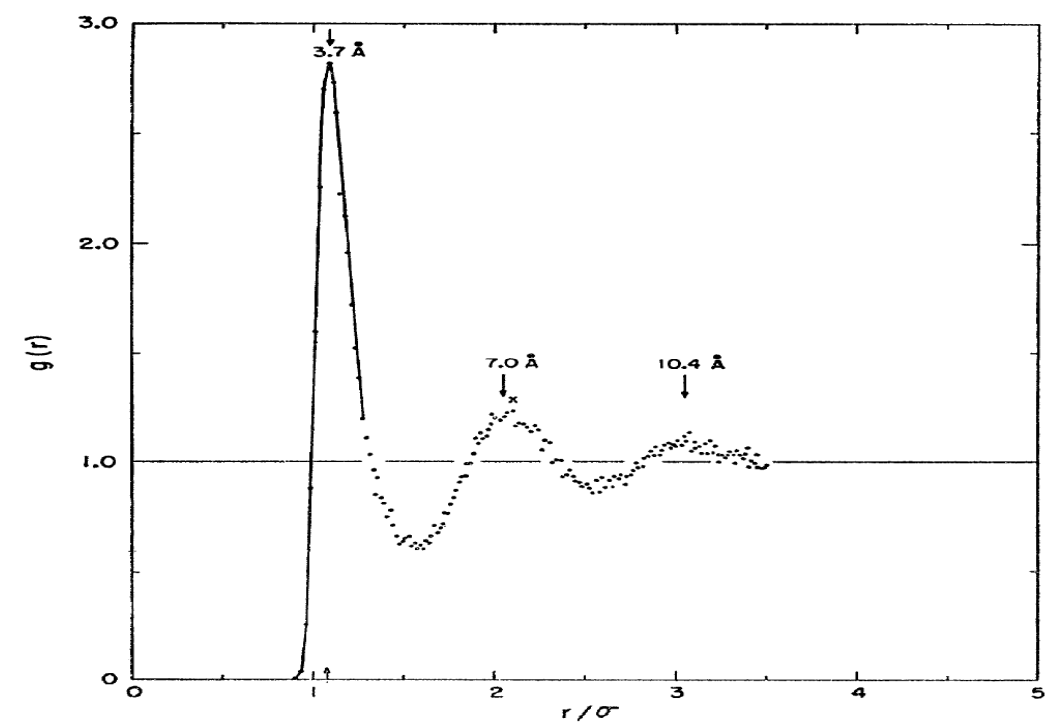

Figure 1 - The radial distribution function in liquid argon [3], T=94.4 K, $\rho=1.374 \mathrm{r} / \mathrm{cm}^{3}$.

The $\mathrm{x}$-axis is normalized in units of $\sigma$ - the parameter of interatomic Lennard-Jones potential. 
The calculated radial distribution function with the help of the written code of molecular dynamics has the form:

$\mathrm{g}(\mathrm{r})$

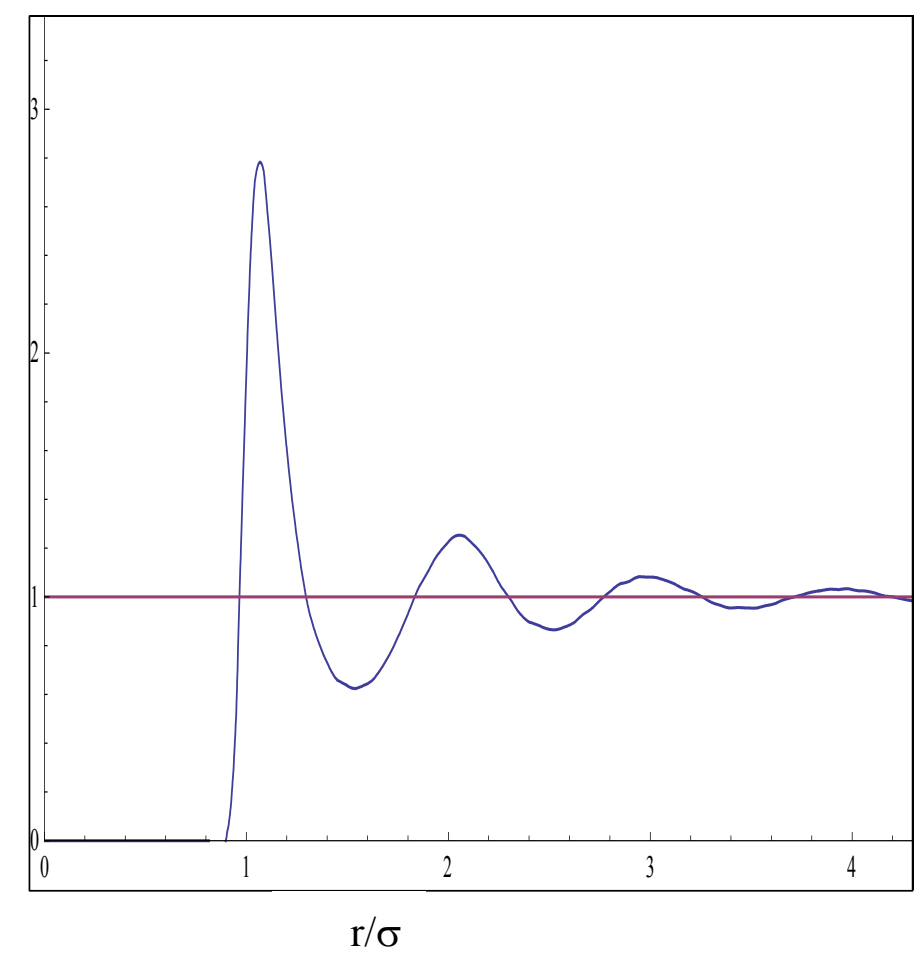

Figure 2 - The radial distribution function in liquid argon, $T=94.4 \mathrm{~K}, \rho=1.374 \mathrm{r} / \mathrm{cm}^{3}$.

The $\mathrm{x}$-axis is normalized in units of $\sigma$ - the parameter of interatomic Lennard-Jones potential.

As we can see from Figures 1 and 2, the obtained radial distribution function is similar to the radial distribution function obtained in [3], which indicates the correctness of the written code for molecular dynamics method based on the proposed criteria.

\section{Static Structural Properties of Strongly} Coupled Plasma

We studied static properties of single-particle plasma for various values of coupling parameter
$\Gamma=\frac{e^{2}}{a k_{B} T}$, here $a=\sqrt[3]{3 / 4 \pi n}$ is the average distance between particles. In this research we used Coulomb potential for description of particles motion. The results given in Figure 3 show that the larger coupling parameter $\Gamma$ the more explicit short range symmetries appear in such plasma. This is in good agreement with the fact that the coupling parameter describes the ratio of the average potential energy of ions to their thermal energy. 


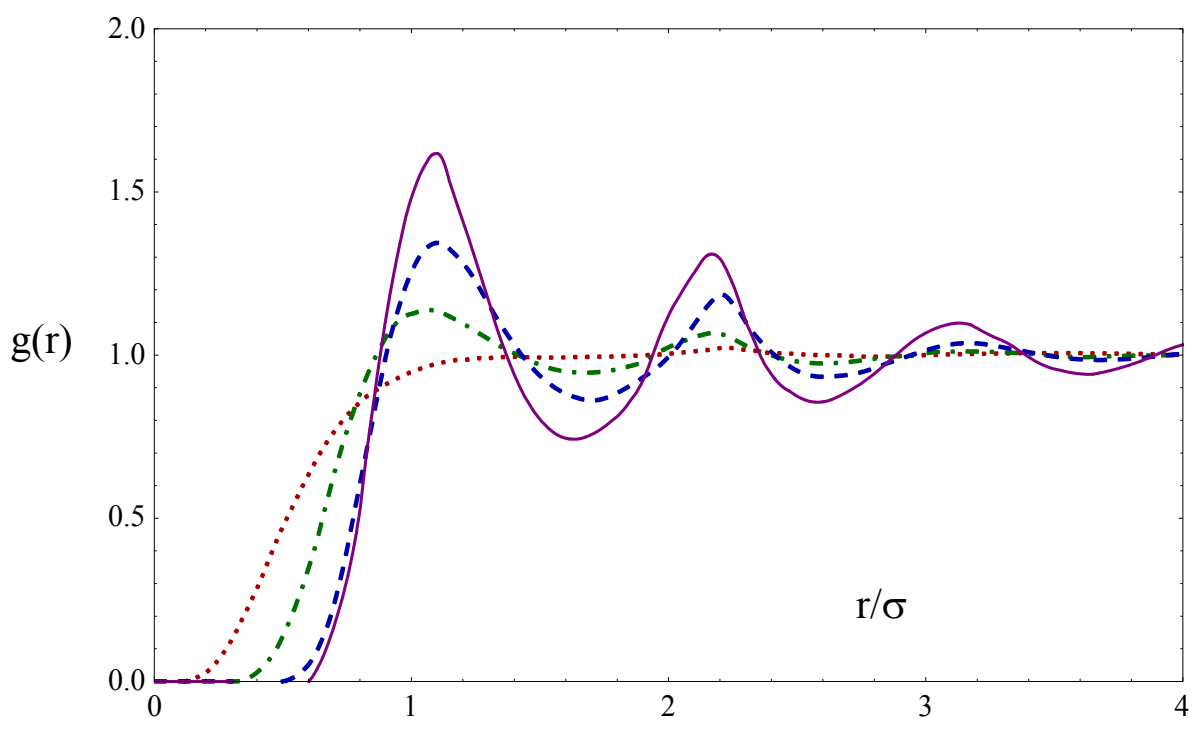

Figure 3 - The radial distribution function of single-particle plasma for various values of the coupling parameter: dotted red line $\Gamma=1$, dot-dashed green line $\Gamma=5$, dashed blue line $\Gamma=10$, solid purple line $\Gamma=20$.

\section{Conclusion}

In this paper we defined the criteria for successfull implementation of molecular dynamics code for studying plasma medium. The code was written in software environment Wolfram Mathematica. It is shown that created code is in good agreement with the results of other researchers. With the help of created code of molecular dynamics in Wolfram Mathematica we calculated radial distribution functions of a dense plasma medium. The calculated radial distribution functions demonstrate that short range symmetries appear in strongly coupled plasma. As our research show the larger coupling parameter $\Gamma$ the more explicit short range symmetries appear in coulomb plasma.

Next possible step of implementaion of the obtained code is calculation of both static and dynamic structural factors on the basis of which it will be possible to construct a Nevanlinna function in the method of moments for a more accurate description of dynamic characteristics of plasma.

\section{References}

1. Allen M.P. and Tildesley D.J., Computer Simulation of Liquids, Oxford: Clarendon Press, 2002.

2. Andersen H.C., Berens P.H., Wilson K.R. A computer simulation method for the calculation of equilibrium constants for the formation of physical clusters of molecules: Application to small water clusters // The Journal of Chemical Physics. - Vol. 76. - N. 1. - P. 648.

3. Rahman A., Correlations in the Motion of Atoms in Liquid Argon, Phys.Rev. - 1964 - Vol. 136 - N2A, -P. 405-411.

4. Baimbetov F.B., Ramazanov T.S. Matematicheskoe modelirovanie $\mathrm{v}$ fizike neidealnoy plazmyi. - Almatyi: Ghylym, 1994. - 212 s. 